\title{
The cautionary tale of side effects of chronic Notch1 inhibition
}

\author{
Sandra W. Ryeom
}

Department of Cancer Biology, Abramson Family Cancer Research Institute, University of Pennsylvania School of Medicine, Philadelphia, Pennsylvania, USA.

\begin{abstract}
Aberrant Notch1 signaling is implicated in several types of cancer. Therefore, Notch signaling pathways are important anticancer targets. Pan-Notch receptor inhibition is associated with numerous complications; thus, selective Notch receptor inhibition has been pursued. Studies have shown minimal side effects with short-term blockade of either Notch1 or its ligand Delta-like 4, but long-term side effects were not investigated. In this issue of the JCI, Liu et al. use mouse models to demonstrate the consequence of long-term Notch 1 inhibition. They present evidence that chronic Notch1 inhibition leads to vascular tumors in the liver and decreased survival, which suggests that Notch1 therapies should be reevaluated.
\end{abstract}

Dysregulation of signaling by members of the Notch family of receptors has been observed in numerous diseases. Activating Notch1 mutations in $\mathrm{T}$ cell acute lymphoblastic leukemia (T-ALL) were identified a number of years ago (1); more recently, Notch1 has been implicated in pancreatic and lung cancer $(2,3)$. Consistent with these observations, deletion of Notch 1 in mice in the context of an activated $K$-ras oncogene leads to accelerated pancreatic intraepithelial neoplasia formation in a mouse model of pancreatic ductal adenocarcinoma (2), and Notch1 expression in patients with non-small cell lung cancer has been shown to be a predictor of poor overall survival (3). These and other recent studies (4) have generated interest in Notch1 as an anticancer target. In this context, a study in which ligand activation of Notch1 was prevented suggested that functional inhibition of Notch1 blocks tumor angiogenesis by triggering nonproductive angiogenesis, the formation of highly disorganized tumor vasculature insufficient to deliver blood and nutrients to tumor cells (5). Thus, inhibition of Notch1 may disrupt both tumor cell proliferation and tumor angiogenesis.

\section{Inhibiting Notch signaling}

The most well-known pan-Notch receptor inhibitors are $\gamma$-secretase inhibitors (GSIs). This class of drugs prevents cleavage of

Conflict of interest: The author has declared that no conflict of interest exists.

Citation for this article: J Clin Invest. 2011; 121(2):508-509. doi:10.1172/JCI45976. which is necessary for transactivation of Notch targets (Figure 1 and ref. 6). However, recent data have indicated that different Notch family members carry out different and sometimes opposing functions in the same tissue and/or cell type (4). Indeed, Notch1 has been shown to function as both an oncogene and a tumor suppressor, depending on the context (7). Thus, it is not surprising that GSIs have been associated with substantial complications in patients, ranging from increased incidence of skin cancer to intestinal toxicity from goblet cell metaplasia caused by inhibition of all four Notch family members (8).

Recently, Siebel and colleagues generated two antibodies that specifically inhibited either Notch1 or Notch2 (9). These Notch1- and Notch2-specific antibodies stabilize the extracellular juxtamembrane negative regulatory region of Notch1 and Notch2, respectively, preventing cleavage of the intracellular domain, even in the presence of their ligands. These antibodies were selected for their ability to specifically inhibit both human and mouse orthologs of either Notch1 or Notch 2 with high affinity. The antibodies demonstrated dosedependent inhibition of either Notch1 or Notch2 signaling in vitro and high specificity for their respective Notch receptor. However, not surprisingly, treatment with the Notch1-specific antibody led to a substantial decrease in $\mathrm{CD} 4^{+}$and $\mathrm{CD} 8^{+} \mathrm{T}$ cells, due to its previously demonstrated role in $\mathrm{T}$ cell development (10). Siebel and colleagues used their Notch1 antibody (9) to treat a T-ALL cell line containing activating the Notch receptor intracellular domain,
Notch1 mutations. Their data demonstrate that use of their Notch1-specific antibody inhibited T-ALL growth in vitro and in xenograft models in vivo. Treatment of xenografted tumors that lacked an activating Notch1 mutation with the Notch1-specific antibody also demonstrated suppression of tumor growth caused by the disruption of tumor angiogenesis (9). Siebel and colleagues took advantage of the highly specific Notch 1 and Notch2 inhibitory antibodies generated in this study to parse out side effects resulting from inhibition of specific receptors. While demonstrating that their Notch1-specific antibody was sufficient to suppress tumor growth in xenograft models of T-ALL, colon carcinoma, and lung carcinoma, only mild goblet cell metaplasia was identified in the intestinal crypts in the presence of Notch1 inhibition alone (9), as opposed to the severe metaplasia observed upon pan-Notch receptor inhibition (8). However, in the work by Siebel and colleagues, antibody-mediated Notch1 inhibition was only examined over a relatively short period of 2-3 weeks; the long-term consequences of Notch1 inhibition were not investigated (9).

In contrast, recent work by Yan et al. examined the effects of functional Notch 1 inhibition by targeting its ligand Delta-like 4 (DLL4; ref. 11). These studies revealed substantial pathologic changes in the liver after 8 weeks of treatment with a DLL4specific antibody in multiple species from rats to monkeys. Endothelial-specific genes known to be important for different aspects of endothelial activation were upregulated in the liver after DLL4 blockade, implicating a role for DLL4-Notch1 signaling in maintaining the liver endothelium in a quiescent state. Furthermore, a subset of rats treated for 8 weeks with the DLL4-specific antibody developed subcutaneous vascular neoplasms in a dose-dependent manner, which suggests that systemic inhibition of Notch1 signaling may disrupt normal endothelial cell homeostasis leading to vascular tumors. However, effects on other organ-specific vascular beds were not explored in this work. 


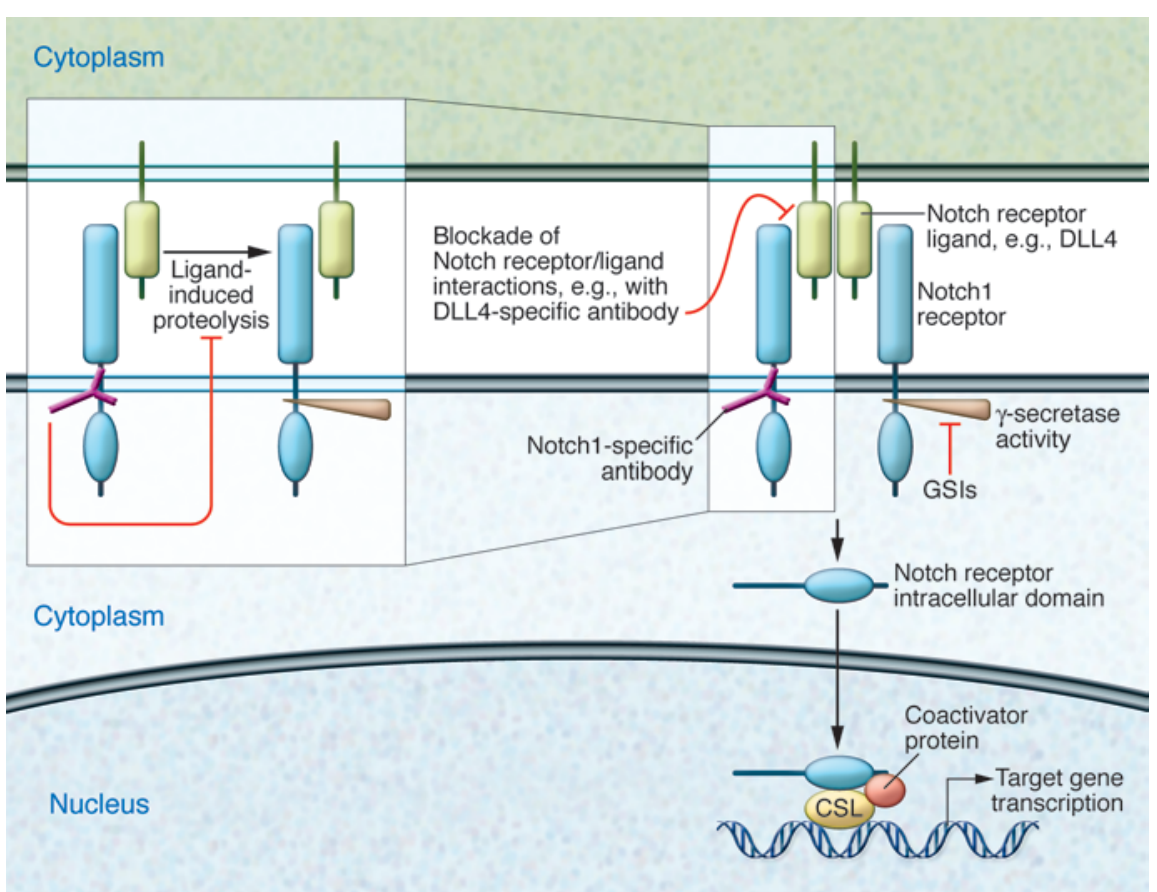

\section{Chronic Notch1 inhibition}

In this issue of the JCI, Liu and colleagues examined the consequences of chronic Notch1 inhibition (12). They used elegant genetics approaches and sophisticated reporter methods in mice to delete and detect Notch1 in tissues in which it is repeatedly activated in a physiologically relevant manner. The ingenuity of this genetic model resides in its ability to model sporadic loss of Notch1 heterozygosity, in which graded deletion of Notch1 occurs over time. Since the previously described studies implicated a role for Notch1 in suppressing endothelial cell activation $(9,11)$, in this work, Liu et al. were able to dissect out which vascular beds were most reliant upon Notch1 signaling (12). They found decreased survival by almost 200 days of mutant mice that lost Notch1 over time, compared with either those mice that remained heterozygous for Notch1 or wild-type littermate controls. Necropsy analysis of numerous organs in the mutant mice revealed that Notch1 was activated most frequently in the liver endothelium, and detailed histopathologic examination of the liver revealed the presence of benign tumors of endothelial cell origin (also known as hemangiomas) in greater than $80 \%$ of the mice. BrdU labeling, which allows for the detection of cycling cells, was found exclusively in Notch1 $1^{-/-}$liver endothelial cells, which indicated that the liver endothelium had a hyperproliferative phenotype in the absence of Notch 1 signaling.

\section{Reevaluating Notch1 therapies}

There is significant interest in targeting the Notch 1 signaling pathways for the treatment of cancer, either by inhibiting Notch 1 receptor activation directly or by sequestering its activating ligands, such as DLL4 (Figure 1). The current work by Liu et al. (12) is of particular interest in this context, as it indicates that the use of long-term anti-Notch 1 therapies needs to be carefully reevaluated. This work also offers some intriguing clues regarding the specificity of Notch 1 activation in tissue-specific vascular beds. Although the authors propose that the increased Notch 1 activation in the liver vasculature may be due to higher remodeling rates in this organ, further work is necessary to explore the mechanism by which Notch 1 signaling promotes endothelial cell homeostasis in different organ environments. Finally, this work offers insight into the molecular mechanisms and signaling pathways that may underlie the formation of benign liver hemangiomas as well as vascular tumors in general.

\section{Acknowledgments}

This work was supported by NIH grant R01CA552679.

Address correspondence to: Sandra W. Ryeom, Department of Cancer Biology, University of Pennsylvania School of Medicine,

\section{Figure 1}

Notch receptor inhibition by various mechanisms. GSIs block $\gamma$-secretase activity, which is necessary to cleave the Notch intracellular domain. Antibodies that bind to Notch ligands such as DLL4 prevent Notch receptor interaction with its ligand. Inhibitory Notch1-specific antibody prevents protease cleavage of the negative regulatory region of Notch1 after ligand activation.
Abramson Family Cancer Research Institute, 311 BRB, 421 Curie Boulevard, Philadelphia, Pennsylvania 19106, USA. Phone: 215.573.5857; Fax: 215.573.2014; E-mail: sryeom@upenn.edu.

1. Aster JC, Pear WS, Blacklow SC. Notch signaling in leukemia. Annu Rev Pathol. 2008;3:587-613.

2. Hanlon L, et al. Notch1 functions as a tumor suppressor in a model of K-ras-induced pancreatic ductal adenocarcinoma. Cancer Res. 2010;70(11):4280-4286.

3. Donnem T, Andersen S, Al-Shibli K, Al-Saad S, Busund LT, Bremnes RM. Prognostic impact of Notch ligands and receptors in non-small cell lung cancer: co-expression of Notch-1 and vascular endothelial growth factor-A predicts poor survival. Cancer. 2010;116(24):5676-5685.

4. Yin L, Velazquez OC, Liu ZJ. Notch signaling: emerging molecular targets for cancer therapy. Biochem Pharmacol. 2010;80(5):690-701.

5. Ridgway J, et al. Inhibition of Dll4 signalling inhibits tumour growth by deregulating angiogenesis. Nature. 2006;444(7122):1083-1087.

6. Shih IeM, Wang TL. Notch signaling, gammasecretase inhibitors, and cancer therapy. Cancer Res. 2007;67(5):1879-1882.

7. Radtke F, Raj K. The role of Notch in tumorigenesis: oncogene or tumour suppressor? Nat Rev Cancer. 2003;3(10):756-767.

8. van Es JH, et al. Notch/gamma-secretase inhibition turns proliferative cells in intestinal crypts and adenomas into goblet cells. Nature. 2005; 435(7044):959-963.

9. Wu Y, et al. Therapeutic antibody targeting of individual Notch receptors. Nature. 2010; 464(7291):1052-1057.

10. Radtke F, Wilson A, Mancini SJ, MacDonald HR. Notch regulation of lymphocyte development and function. Nat Immunol. 2004;5(3):247-253.

11. Yan M, et al. Chronic DLL4 blockade induces vascular neoplasms. Nature. 2010;463(7282):E6-E7.

12. Liu Z, et al. Notch1 loss of heterozygosity causes vascular tumors and lethal hemorrhage in mice. J Clin Invest. 2011;121(2):800-808. 